\title{
Performance Comparison of Text Based Game Prototypes Using GTmetrix
}

\author{
Ida Bagus Kerthyayana Manuaba \\ Computer Science Department, School of Computer Science, \\ Bina Nusantara University, \\ Jakarta, Indonesia 11480 \\ imanuaba@binus.edu
}

\begin{abstract}
Access to the internet and the advanced of technology allow people to experience in playing a game, not limited to their game console or through a PC, but also through their gadget like mobile-phones. Based on our initial study, a text-based game prototype has shown its potentiality as an alternative media to enhance youth literacy. The prototype has been developed in webbased platform that allow users to play this game through their mobile-phone. This paper describes an extend study to explore on how well the performance of text- based game prototype, when it was run online through a mobile device. For this purpose, a testing tool, named "GTmetrix" is used to review the performance of mobile device that run two different text-based game prototype platforms, which are Quest and Ink Platforms. For further comparison, this paper also included additional test scenario to see the performance of these two prototype platforms through a desktop device. These testing was conducted through different mobile network speeds (2G/3G/4G). Two different test approaches, PageSpeed and YSlow, were used to analyze the performance. Based on the results, it can be concluded that both prototype platforms are still compatible to be played through mobile devices, with Ink platform perform slightly better compare to the Quest platform in several test setting.
\end{abstract}

Keywords-Text-based Game, Mobile Device, Performance Test

\section{INTRODUCTION}

Despite of the advance technology successfully brings transformation of reading appearance, the people interest of reading in Indonesia still low. It is showed based on the observation of 5th grade elementary school students in Bandung Regency that had low reading comprehension ability for informational text, which can be seen from their low ability to express explicit meanings, define the main idea, search for keywords and retell the contents of the text with their own words [1]. The awareness of people to read, especially for youth generation, need a lot of improvement. The encouraging from closest environments, such as family or school, still insufficient to engage youth generation to do reading activity.

In order to find alternative tools in improving youth literacy in Indonesia, our previous study [2] has been demonstrated that text-based game using Interaction Fiction approach has a potential for helping people to know and understand a literature, especially for Indonesia's folklore story as the test scenario. Our further study also successfully confirmed the eminence of Interactive Fiction approach compare to Narrative Fiction (or traditional book) in terms of reader's understanding, acquired moral value, and user engagement [3]. Based on that study results, we continue our study further to see other potentiality of text-based game prototype with Interactive Fiction approach.

Based on survey data by Pew Research Centre [4] showed that smartphone ownership rates in emerging and developing countries such as Indonesia are rising at an outstanding percentage. It is ascending from a median of $21 \%$ in 2013 to $37 \%$ in 2015 , and still continually growing in the following years. Additionally, in most of countries, youth generation and millennials (those ages 18 to 34) are much more likely to be internet and smartphone users compared to those ages 35 and above. Youth generation and millennials also tend to access the internet in daily basis and massively active in social media at higher rates compare to their older counterparts. Hence, this trends showed that there is shifting transformation of traditional tools into a digital form. Most people today, especially youth generation and millennials gain information paperless, directly from their smartphone.

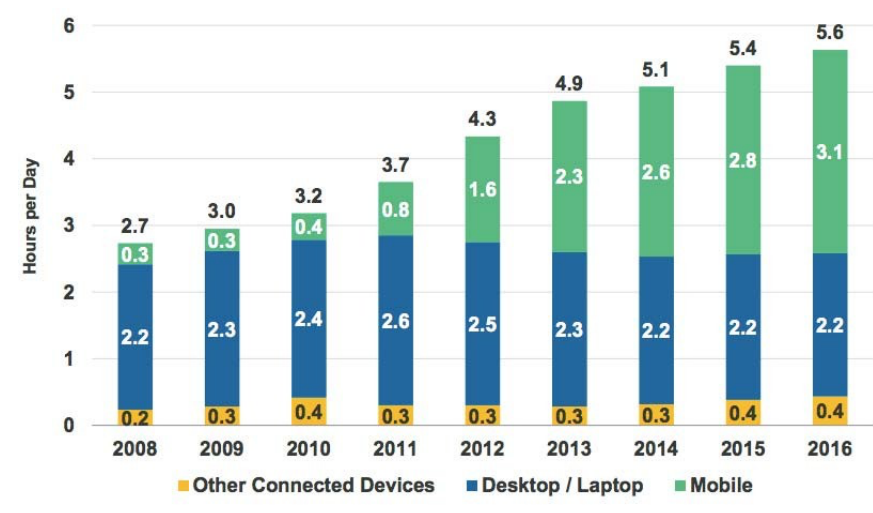

Fig. 1. Time Spent Per Adult User per Day with Digital Media, USA, $2008-2016[5]$.

As the impact of current advanced of technology, people use their mobile phone and tablet more often than Personal Computer. Based on Fig. 1 above on the example data survey of the time spent per user per day with digital media in USA from 2008 to 2016[5], it shows that there is a trend to substitute the usage of Desktop/Laptop by Smartphone (Mobile) in the future since more people do almost everything from their gadget, especially smartphone. Hence, smartphone becomes a potential device to be used not only limited to a communication device, but also for other purposes such as entertainment, education and any other medias. Based on those data, applying text-based game 
in smartphone become a good alternative to enhance the usage of the prototype application, especially when we are aiming youth generations and millennials as the target users of the game application.

As part of our continuation study, this paper is discussing the performance test which focused on the comparison of prototype's performance through mobile-phone and desktop. Since the text-based game prototype has been deployed online in two different platforms, in Quest [6] and Ink [7] platforms, the performance test was conducted using these two platforms. GTmetrix [8] with PageSpeed and Yslow test approaches has been chosen as the performance testing tool. Waterfall Graph is also used to represent the performance results of those two prototype platforms.

\section{TESTING TOOLs: GTMETRIX}

Based on data by Hitwise's report [9] mentioned that 50$65 \%$ of google search is coming from mobile devices. The report also mentioned that people are five times more likely to leave a mobile site if its not user-friendly. In addition, the report also stated that around half of visitors of a webpage will leave if a page does not load within 3 seconds. Hence, it is necessary to see how fast could a web application could be load through mobile device.

In using a mobile-device as alternative to desktop/laptop for running text based game application, the network speed is also an important factor that must be counted into a consideration since mobile network speed $(2 \mathrm{G} / 3 \mathrm{G} / 4 \mathrm{G})$ is not as faster as broadband/fiber internet. Here, there is possibility that the web page may load quicker on a desktop but slower on mobile devices. Hence, performing a test to check how well this game could be load from mobile devices in different mobile network speed is also necessary.

In order to perform the performance test, choosing an appropriate testing tool is an important step to be done. According to an article from GeekFlare [10], there are number of test tools that could be used for checking how fast a web application could be load from mobile devices. The list of tools is SiteRelic, Mobitest, Think with Google, GTmetrix, SynTraffic and DotcomMonitor.

Each testing tool has its own advantages and disadvantages. However, this paper will not discuss further detail about the advantages and disadvantages of each tool. This paper only focuses on one of the testing tools, named GTmetrix, which has the closest features required to the aims of this study.

GTmetrix is one of the most popular web page performance testing tools both for desktop and mobile version [8]. the GTmetrix has modern Graphical User Interface that very easy and intuitive to be used. It looks very promising and it could also give a recommendation how to improve the performance as the feedback result..

GTmetrix also allows to perform the test using different browser such as Chrome and Firefox browsers. It can be set on different devices, such as mobile devices or desktop/laptop. It could also be used with different setting of mobile network speed $(2 \mathrm{G} / 3 \mathrm{G} / 4 \mathrm{G})$ and modified test server location. It costs free, but when we registered and login, we could save the performance result and enhance with comparison from different setting scenarios.

By using GTmetrix tool, the performance metrics is measured using PageSpeed and YSlow. PageSpeed is a measurement by Google to see on how fast the content of the page is load [11]. While Yslow test is a test that analyses web pages and why they are slow based on Yahoo!s rules for high performance web sites [12]. For performance testing indicators, variable used for PageSpeed and Yslow are listed in Table 1 below:

TABLE I. INDICATORS TESTED FOR PERFORMANCE TEST OF $P A G E S P E E D$ AND YSLOW TEST.

\begin{tabular}{|c|c|}
\hline PageSpeed & Yslow \\
\hline $\begin{array}{l}\text { Avoid a character set in the meta } \\
\text { tag }\end{array}$ & Add Expires headers \\
\hline Avoid bad requests & Avoid AlphaImageLoader filter \\
\hline Avoid CSS@import & Avoid CSS expressions \\
\hline Avoid landing page redirects & Avoid HTTP 404 (Not Found) error \\
\hline Combine images using CSS sprites & Avoid URL redirects \\
\hline Defer parsing of JavaScript & Compress components with gzip \\
\hline Enable gzip compression & Configure entity tags (ETags) \\
\hline Enable Keep-Alive & Make AJAX cacheable \\
\hline Inline small CSS & Make favicon small and cacheable \\
\hline Inline small JavaScript & Make fewer HTTP requests \\
\hline Leverage browser caching & Make JavaScript and CSS external \\
\hline Minify CSS & Minify JavaScript and CSS \\
\hline Minify HTML & Reduce cookie size \\
\hline Minify JavaScript & Reduce DNS lookups \\
\hline Minimize redirects & Reduce DOM elements \\
\hline Minimize request size & Remove duplicate JS and CSS \\
\hline Optimize images & Use a Content Delivery Network \\
\hline $\begin{array}{l}\text { Optimize the order of styles and } \\
\text { scripts }\end{array}$ & Use cookie-free domains \\
\hline Prefer asynchronous resources & Use GET for AJAX requests \\
\hline Put CSS in the document head & \\
\hline $\begin{array}{l}\text { Remove query strings from static } \\
\text { resources }\end{array}$ & \\
\hline $\begin{array}{l}\text { Serve resources from a consistent } \\
\text { URL }\end{array}$ & \\
\hline Serve scaled images & \\
\hline Specify a cache validator & \\
\hline Specify a character set early & \\
\hline $\begin{array}{l}\text { Specify a Vary: Accept-Encoding } \\
\text { header }\end{array}$ & \\
\hline Specify image dimensions & \\
\hline
\end{tabular}

In addition to the two performance metric indicators, GTmetrix also performs the test to check Time taken to load, Page size, Number of requests using Waterfall graph. The Waterfall is one of useful tools for pinpointing bottleneck of website's speed. The steps that each asset can go through the test are as follows time to resolve the DNS (DNS Lookup), time taken to create a connection (Connecting), time spent in the browser queue waiting for connection (Blocking), time taken to send a request (Sending), time spent waiting for the response (Waiting), and time taken to download the web content (receiving).

\section{Methodology}

The methodology used for analyzing this performance testing is a Quantitative method. Quantitative data allows a direct assessment of the performance result based on PageSpeed, YSlow indicators and Waterfall graph. Based on 
previous study [2][3], in order to develop text-based game prototype, two different platforms were used which are Quest platform and Ink platform. In this study, the data is collected based on these two prototype platforms performance to analyze on how fast the page could be loaded. Next is the detail setup and testing scenario for the testing:

\section{A. Setup}

For this testing setup, there are combinations of elements were tested using GTmetrix tool. Detail of the elements tested and the descriptions could be seen in Table II below.

TABLE II. ELEMENTS TESTED AND DESCRIPTION

\begin{tabular}{|c|c|c|}
\hline No & Elements & Descriptions \\
\hline 1 & $\begin{array}{l}\text { Prototype } \\
\text { URL tested }\end{array}$ & $\begin{array}{l}\text { Quest Platform } \\
\text { http://play2.textadventures.co.uk/Play.aspx?id=ed } \\
\text { itor/568c39ff-4c99-4d9a-acd6- } \\
\text { 646fe85d6f11\%2fPutri+Pinang+Masak.aslx } \\
\text { Ink Platform } \\
\text { https://if.gamelabnetwork.com/interactive.html }\end{array}$ \\
\hline 2 & Platform & $\begin{array}{l}\text { Desktop and Android (Galaxy Nexus } \\
\text { Specification) }\end{array}$ \\
\hline 3 & $\begin{array}{l}\text { Server test } \\
\text { location }\end{array}$ & Vancouver Canada ${ }^{\mathrm{a}}$ \\
\hline 4 & Connection & $\begin{array}{l}\text { Un-Throttled Connection } \\
\text { Throttle Connection (2G/3G/4G(LTE)) }\end{array}$ \\
\hline 5 & Browser & $\begin{array}{l}\text { Chrome and Firefox (desktop) and NativeBrowser } \\
\text { (Mobile) }\end{array}$ \\
\hline
\end{tabular}

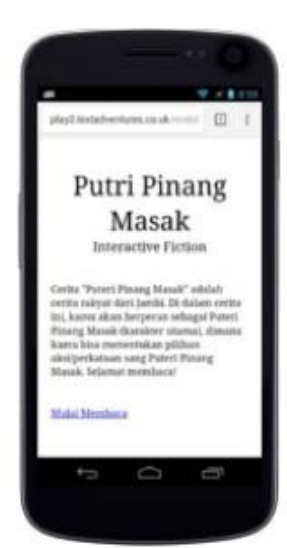

${ }^{\text {a. }}$ Being used as a default server's location

Fig. 2. Prototypes run on Galaxy Nexus.

Since the text-based game prototype was build based on wed based application. It could be played in any devices that support web browser. For this test scenario, this game prototype is tested using Android smartphone Galaxy Nexus [13]. Fig. 2 shows the two text-based game prototype platforms run on the Galaxy Nexus.

\section{B. Testing Scenario}

In order to perform the performance test for two different game prototype platforms, below are the scenario for the testing:
1. The text-based game prototype using two different platforms were tested in desktop environment using google chrome browser. In this testing, three different mobile network (throttle connection) in $2 \mathrm{G}, 3 \mathrm{G}$ and $4 \mathrm{G}$ (LTE) speed connections were tested.

2. For further result, testing these two platforms were also conducted through different type of browsers, which were Mozilla Firefox and Google Chrome browsers. In this testing, the connection is set with un-throttled connection

3. As the main objective of this study, to evaluate these two game prototype platforms through mobile devices, these two platforms were also tested using Android mobile phone Galaxy Nexus. The connection for this test is set to un-throttled connection with native browser applications. This scenario could also provide information on how well this game prototype run by mobile device compare to desktop based.

4. In exploring more detail on the test performance on Android mobile, a number of approaches such as PageSpeed test, YSlow test, and Waterfall Graph are used to explore further detail indicators that effected speed performance to load the application page.

5. Summary of overall test performance was described more detail for both game prototype platforms.

\section{RESULT}

Following the scenarios described on testing scenario above, this section describes the result of performance test from these two game prototype using GTmetrix.

First, the data have been classified into several comparison settings. Then from the data in Fig. 3 to Fig. 5, The are showing a comparison of performance test of the game prototype in a desktop setting through $2 \mathrm{G}, 3 \mathrm{G}$ and $4 \mathrm{G}$ (LTE) connections. Based on the data, the total time for loading page for Ink platform load faster than Quest platform.

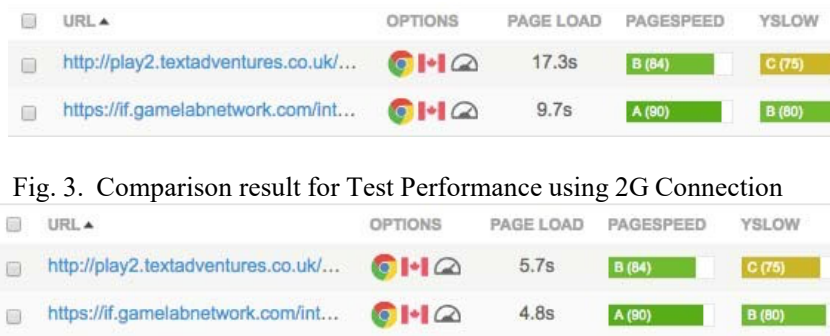

4. Comparison result for Test Performance using 3G Connection 


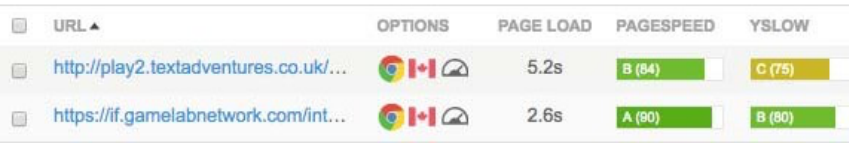

Fig. 5. Comparison result for Test Performance using 4G/LTE Connection

Based on PageSpeed test indicators, the Ink platform is categorized with average A rank $(90 \%)$ but Quest platform is only categorized with average B rank (84\%). Similar to PageSpeed test, Yslow test also shows similar performance, as Ink Platform perform slightly better compare to Quest platform with lower score compare to PageSpeed test, Ink Platform in B rank (80\%) and Quest platform in with C rank (75\%) in all three different connections tested.

Further performance comparison had also been conducted. In this test, the performance of these two game prototype platforms had been compared in two different browsers, Mozilla Firefox and Google Chrome browsers. The data comparison of these two game platforms on different browsers could be seen in Fig 6 .

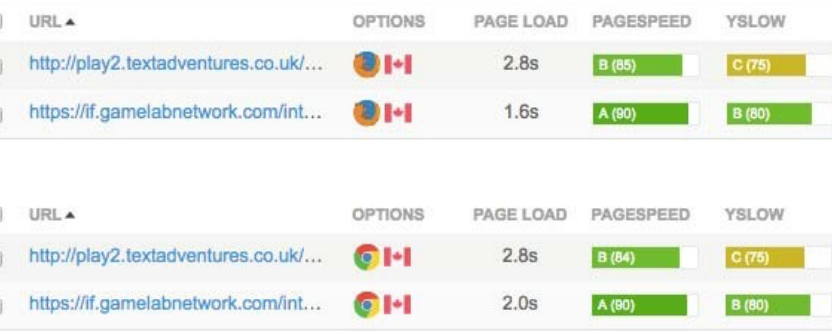

Fig. 6. Comparison result for using Mozilla Firefox and Google Chrome browser

From this Fig 6 also shows that Ink Platform performs better than Quest Platform. Especially in Firefox, the page loading for Ink Platform is $0.4 \mathrm{~s}$ faster compare to when it was loaded using chrome browser.

Further performance comparison test had also been implemented through Android mobile phone Galaxy Nexus. In this testing scenario, the time taken to load the page of these two platforms look almost the same (3.8s). However, based on PageSpeed and Yslow test, the data still shows Ink platform perform better than Quest platform.

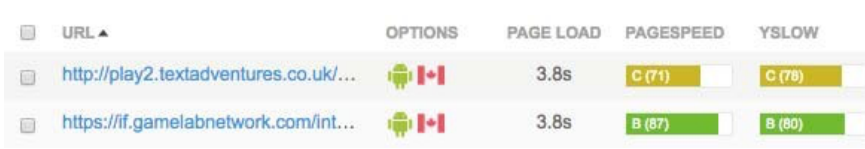

Fig. 7. Comparison result for Test Performance in Android Mobile

Looking for detail data based on the performance testing in Android Mobile devices, below are the data based on PageSpeed (see Table III) and YSlow (see Table IV) test:

TABLE III. Performance Result For PageSPeEd

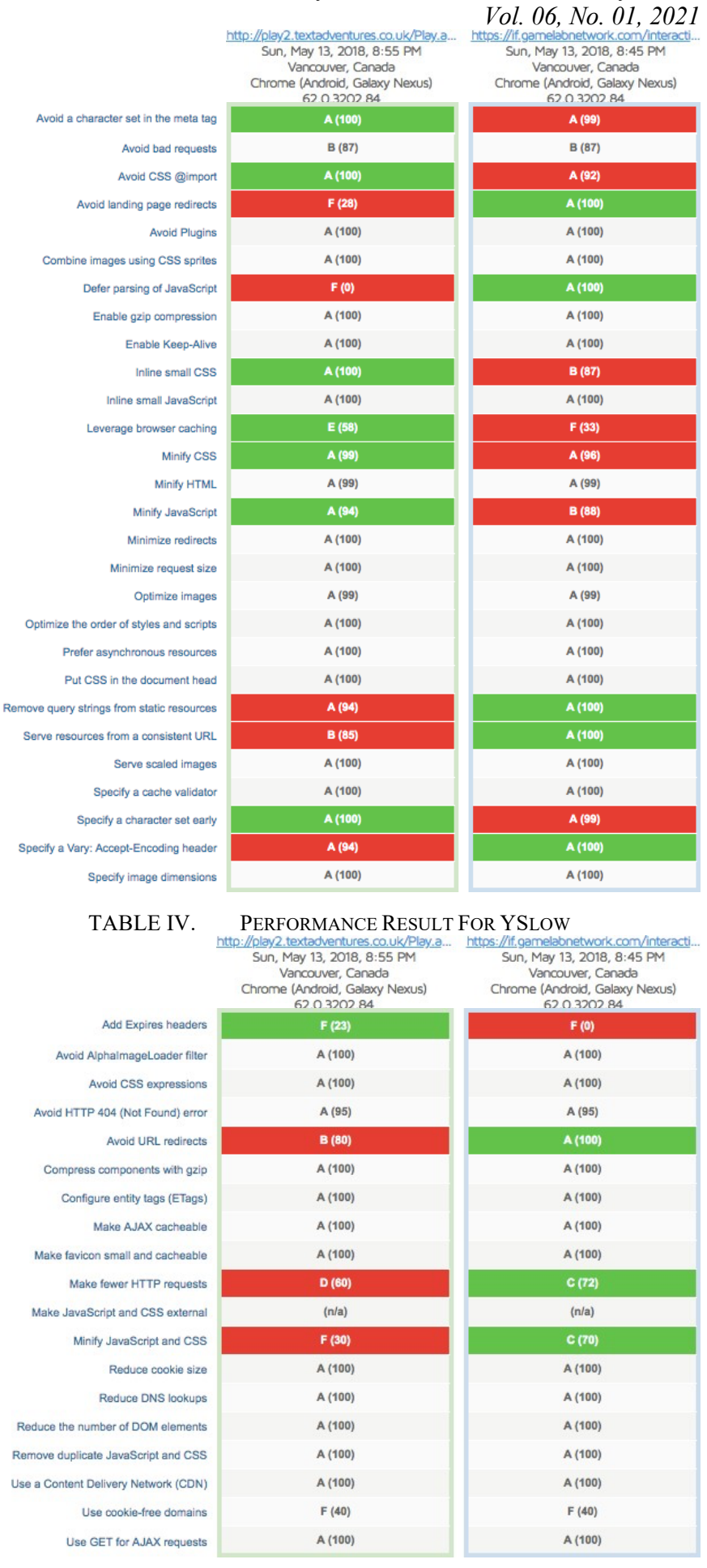

Exploring further with Waterfall graph, Fig 8 and Fig 9 showthe detail time taken to load the the game prototype pages. 


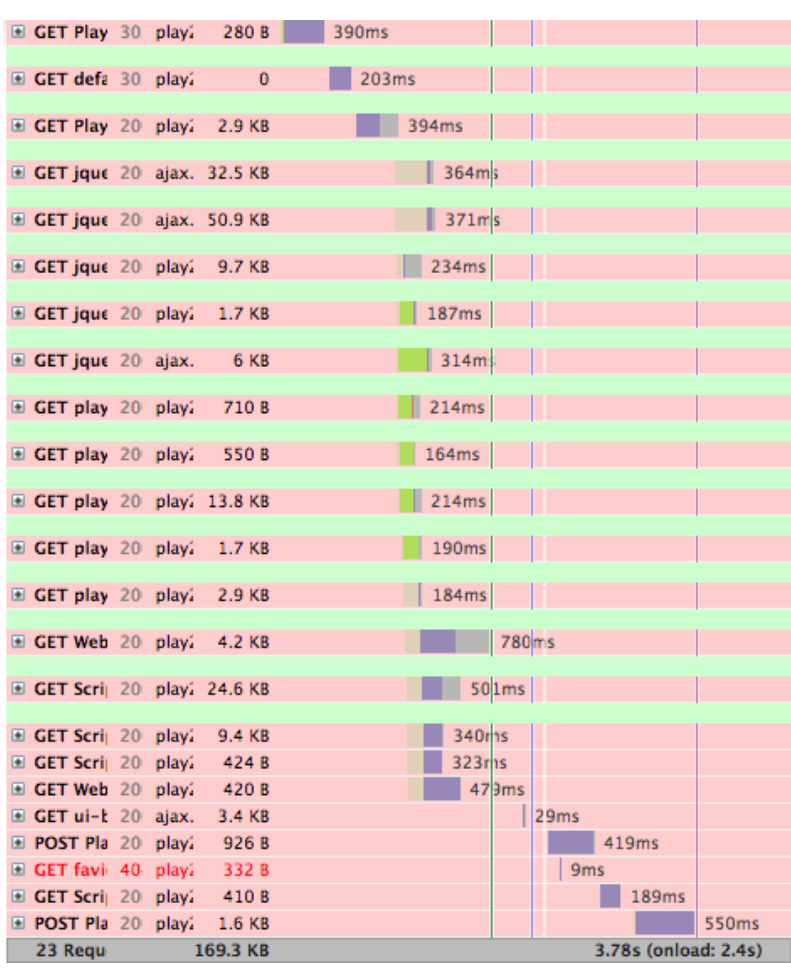

Fig. 8. Waterfall chart on Quest platform page load testing

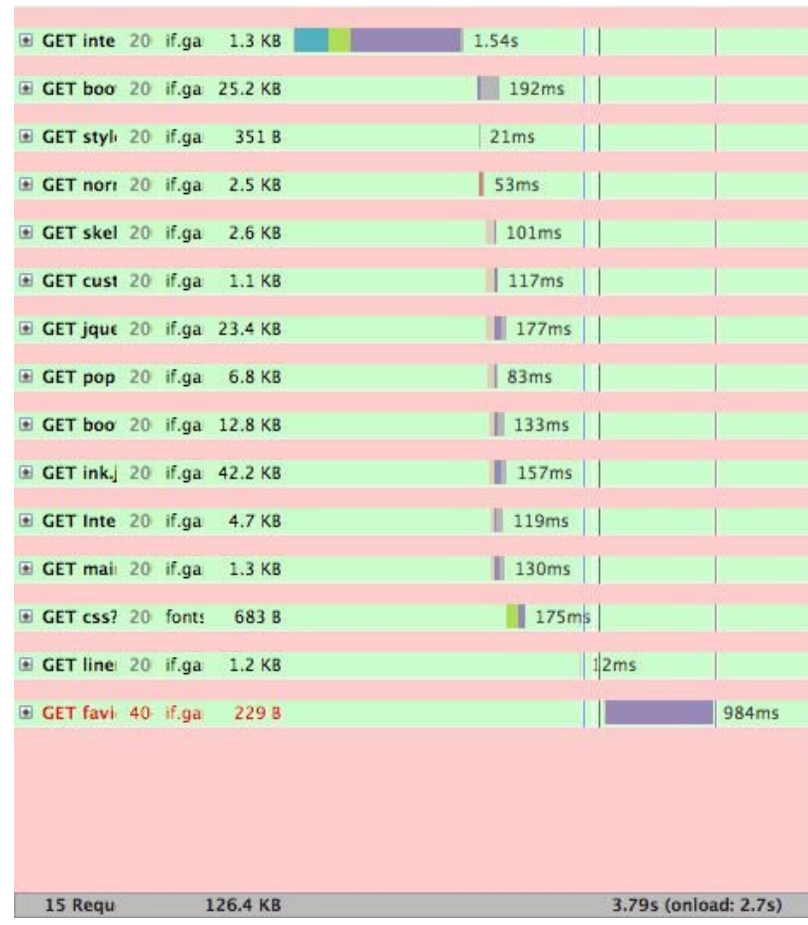

Fig. 9. Waterfall graph on Ink platform page load testing

Based on the data result above it could be summarized throughsummary graphs below:
Vol. 06, No. 01, 2021

I http://play2.textadventures.co.uk/Play.aspx?id=editor/5...

https://if.gamelabnetwork.com/interactive.html PageSpeed Scores

Higher is better

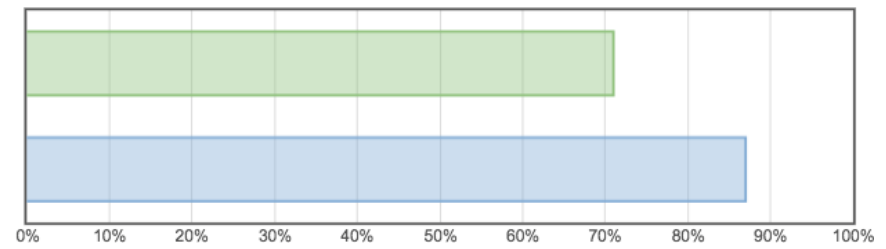

YSlow Scores

Higher is better

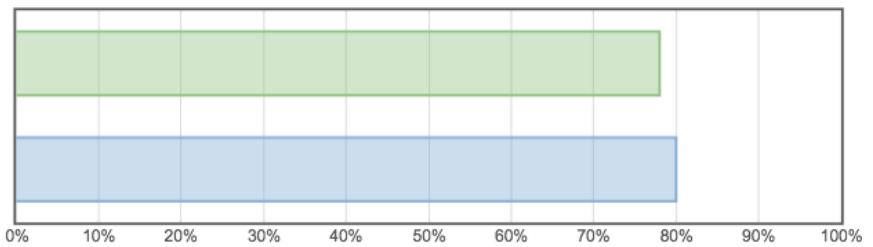

Fig. 10. Summary graph for each method tested

\section{DISCUSSION}

Based on the result mentioned in previous section, performance test especially for using PageSpeed and YSlow test rank showed that Ink platform performs slightly better compare to Quest platform. Based on 27 indicators tested from

\section{Fully Loaded Times}

Lower is better

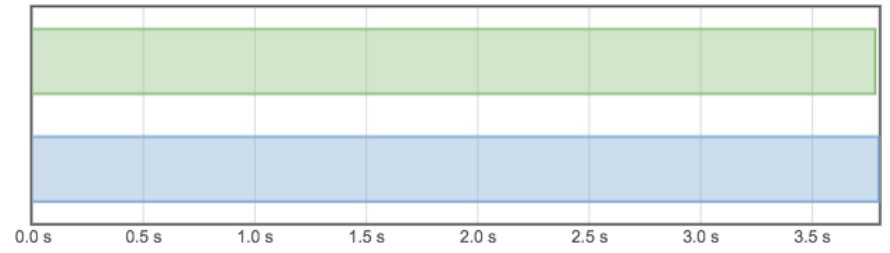

Page Sizes

Lower is usually better

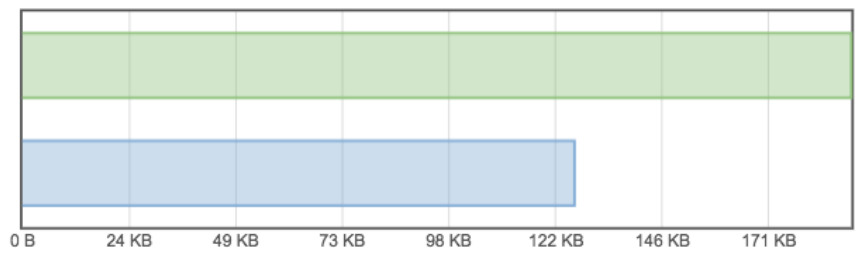

Request Counts

Lower is usually better

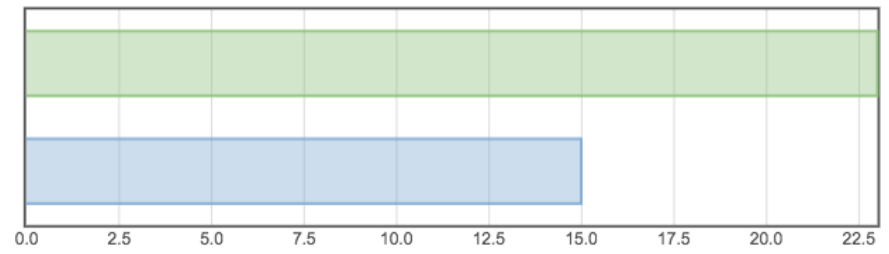


PageSpeed test, there are seven indicators that made Quest are looked more superior compare to Ink platform, however the different are very small. There are five indicators from Ink that perform better than Quest platform, but the different is bigger for these indicators. While the rest of indicators showed equal performance. The big different still showed that Ink platform better than Quest platform. Detail could be seen on Table III above.

Similar to PageSpeed test, in average, the YSlow test also showed Ink platform was better than Quest platform for all different test scenario. Based on 19 indicators tested, 3 indicators in this test showed Ink platform better than Quest platform, but not in header express indicator. The rest of indicators showed equal performance.

Looking for different variable test, for Time taken to load the page, it was definitely affected by the mobile network connection speeds (2G/3G/4G-LTE). For this scenario testing, it showed that Ink platform could be loaded almost twice faster especially using 4G/LTE connections with time to load less than $3 \mathrm{sec}$.

In condition for un-throttled connection, the time taken to load the page for Ink platform still showed faster compare to Quest platform. However, the page on both platforms could be loaded below $3 \mathrm{sec}$. This conditions also occurred when the scenario test was set for different browsers, which Mozilla Firefox perform better compare to Google Chrome.

When the scenario test was set using Galaxy Nexus, the Time taken to load the page became slightly slower compare to the desktop with page load time up to $3.8 \mathrm{sec}$ for both platforms. The result shows that performance in mobile device still manageable for both platforms. However, looking at Waterfall graph at Fig. 8 and Fig. 9, the number of request and page loaded size for Ink platform were smaller compare to Quest platform.

Based on data result of two platforms tested, even though both platforms performance was still acceptable for mobile application testing scenario, Ink platform perform better than Quest platform.

\section{CONCLUSION}

This study has showed performance comparison test between two text-based game platform on mobile device scenario. Using GTmetrix as a tool for testing, this study could implement a number of performance test methods such as PageSpeed and YSlow test rank, and also Waterfall graph test for Time taken to load the application page.

Based on the result and the discussion, it can be concluded that both of text-based game prototype platforms could run satisfactory on mobile device with range time taken for load the page up to $3.8 \mathrm{sec}$. This time could be resulting differently based on the type of connection used and type of browsers.

Even though both platforms could perform well in mobile device tested, Ink platform shows better performance in a number of testing variables compare to Quest platform. Hence, further development of this study could be recommended to focus on the development using Ink platform.

However, further study is still required to enhance the features and also to see on how well this text-based game prototype could be utilized as a media in enhancing Indonesia literacy.

\section{ACKNOWLEDGMENT}

This study is supported by School of Computer Science at BINUS University International. I would like to thank three of my students Albert Darmawan, Ieuan Ignatius and Shan Valdo for their contribution on developing the story and the prototypewith me.

\section{REFERENCES}

[1] T. Indri Hardini, R. Dwi Puspita, and U. Sunan Gunung Djati, "Learning Tasks Design in Improving Reading Comprehension Ability of Informational Text of 5th Grade in Indonesia: An Interactive- compensatory Model Use Rully Agung Yudhiantara," J. Languange Teach. Res., vol. 9, no. 3, pp. 555-560, 2018.

[2] I. B. K. Manuaba, "Text-Based Games as Potential Media for Improving Reading Behaviour in Indonesia," in Procedia Computer Science, 2017, vol. 116.

[3] A. Darmawan, I. Ignatius, S. Valdo, and I. B. K. Manuaba, "A Comparative Study Between Narrative Fiction and Interactive Fiction to Enhance Youth Literacy in Indonesia," in 11th Annual International Conference on Computer Games Multimedia and Allied Technologies (CGAT 2018), 2018.

[4] J. Poushter and R. Stewart, "Smartphone Ownership and Internet Usage Continues to Climb in Emerging Economies But advanced economies still have higher rates of technology use," 2016.

[5] M. Meeker, "Internet Trends 2017 - Code Conference," 2017.

[6] textadventures.co.uk, "Quest - Write text adventure games and interactive stories." [Online].

[Accessed: 22-Jun-2018]

Available: http://textadventures.co.uk/quest

[7] inkle, “ink - inkle's narrative scripting language." [Online]. Available: https:/www.inklestudios.com/ink/. [Accessed: 22-Jun2018].

[8] gtmetrix.com, "GTmetrix | Website Speed and Performance Optimization." [Online]. Available: https://gtmetrix.com/. [Accessed: 22-Jun-2018].

[9] J. Fetto, "What do consumers search for from their smartphones and tablets?"

[10] C. Kumar, "How Fast Your Website Load from Mobile? 'Test Now," Performance, 2018. [Online]. Available: https://geekflare.com/website- load-time-mobile/. [Accessed: 22-Jun$2018]$.

[11] developers.google.com, "Make the Web Faster | Google Developers." [Online]. Available: https://developers.google.com/speed/. [Accessed: 22-Jun-2018].

[12] yslow.org, "YSlow - Official Open Source Project Website." [Online]. Available: http://yslow.org/. [Accessed: 22-Jun-2018].

[13] devicespecifications.com, "Samsung Galaxy Nexus I9250 Specifications." [Online]. Available: https://www.devicespecifications.com/en/model/43b12883. [Accessed: 22-Jun-2018]. 